\title{
Papers
}

\section{Randomised controlled trial of midwife led debriefing to reduce maternal depression after operative childbirth}

\author{
Rhonda Small, Judith Lumley, Lisa Donohue, Anne Potter, Ulla Waldenström
}

\begin{abstract}
Objective To assess the effectiveness of a midwife led debriefing session during the postpartum hospital stay in reducing the prevalence of maternal depression at six months postpartum among women giving birth by caesarean section, forceps, or vacuum extraction.

Design Randomised controlled trial.

Setting Large maternity teaching hospital in

Melbourne, Australia.

Participants 1041 women who had given birth by caesarean section $(n=624)$ or with the use of forceps $(\mathrm{n}=353)$ or vacuum extraction $(\mathrm{n}=64)$.

Main outcome measures Maternal depression (score $\geqslant 13$ on the Edinburgh postnatal depression scale) and overall health status (comparison of mean scores on SF-36 subscales) measured by postal questionnaire at six months postpartum.

Results 917 (88\%) of the women recruited responded to the outcome questionnaire. More women allocated to debriefing scored as depressed six months after birth than women allocated to usual postpartum care $(81(17 \%) v 65(14 \%))$, although this difference was not significant (odds ratio $=1.24,95 \%$ confidence interval 0.87 to 1.77 ). They were also more likely to report that depression had been a problem for them since the birth, but the difference was not significant $(123(28 \%)$ v $94(22 \%)$; odds ratio $=1.37,1.00$ to 1.86$)$. Women allocated to debriefing had poorer health status on seven of the eight SF-36 subscales, although the difference was significant only for role functioning (emotional): mean scores $73.32 v 78.98, t=-2.31$, $95 \%$ confidence interval -10.48 to -0.84 ).

Conclusions Midwife led debriefing after operative birth is ineffective in reducing maternal morbidity at six months postpartum. The possibility that debriefing contributed to emotional health problems for some women cannot be excluded.
\end{abstract}

\section{Introduction}

Operative birth has been associated with considerable maternal morbidity, including depression, guilt, regret, loss of self esteem, prolonged pain, discomfort, infection, grief reactions, feelings of violation, dissatisfaction with care, and occasionally hostility to hospital staff. ${ }^{1-6}$ One intervention recommended to reduce morbidity, particularly psychological morbidity, is debriefing. ${ }^{3}$ Women are given a structured opportunity to discuss their experiences of labour and delivery with an empathic listener shortly after the birth. Debriefing has been recommended as a health promoting strategy for all women after childbirth, in recognition that even an uncomplicated birth can be traumatic ${ }^{78}$ and in the belief that "talking things through" can only prove beneficial. A recent survey of health trusts in England and Wales (response rate 183/211) found that 36\% have formal arrangements in place for debriefing women after childbirth and another $26 \%$ have plans to introduce such an arrangement (S Marchant, L Davidson, and J Garcia, personal communication).

Outside obstetrics, psychological debriefing after traumatic events has been common for many years, despite the paucity of rigorous research evaluating its effectiveness. $^{910}$ Concerns about the potential for debriefing to do harm have been highlighted in the first systematic review, ${ }^{11}$ which not only found no evidence of benefit but also reported significantly increased odds of post-traumatic stress disorder at 13 months in patients with burn trauma who had received debriefing. ${ }^{12}$

Only one trial included in the systematic review was in reproductive medicine. This study found that subsequent emotional adaptation after spontaneous miscarriage was not significantly influenced by debriefing. ${ }^{13}$ Two trials of debriefing after childbirth have been reported: one small trial in the United Kingdom reported positive findings for debriefing, ${ }^{8}$ whereas the other, larger trial from Australia found no positive effect. $^{14}$ The current study arose from an Australian population based survey that found raised rates of depression eight to nine months postpartum in women who had operative deliveries (adjusted odds ratio $=2.03$ ) compared with women who had spontaneous vaginal deliveries. ${ }^{2}$

\section{Participants and methods}

\section{Aims}

The primary aims were to reduce the prevalence of depression by one third (defined as a score of $\geqslant 13$ on the Edinburgh postnatal depression scale ${ }^{15}$ ) six months after the birth, from an expected $24 \%$ in the standard care group ${ }^{2}$ to $16 \%$ in the debriefing group and to improve overall maternal health (as measured by mean scores on the SF-36 health status measure subscales ${ }^{16}$ ).

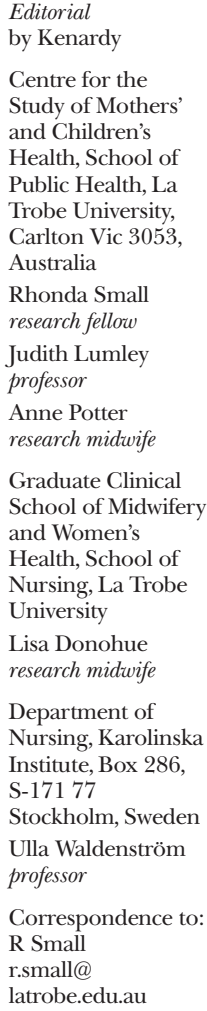

BMJ 2000;321:1043-7

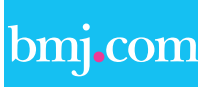

A table showing women's views of their care is available on the $B M J$ 's website 


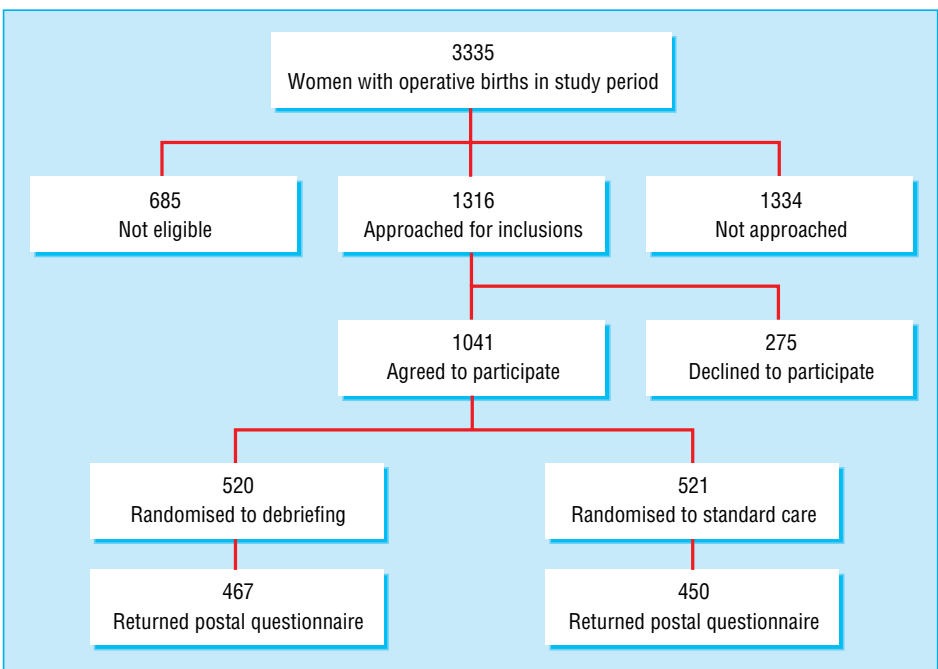

Recruitment and participation of women in the trial

A secondary aim was to reduce dissatisfaction with postpartum hospital care.

\section{Sample size and study power}

The sample size $(\alpha=0.05$, two sided $\beta=0.20)$ required for the primary aim was 416 in each group. We aimed to recruited 1040 women to allow for a $5 \%$ refusal rate and a possible loss to follow up of $20 \%$. This sample size was also more than adequate to detect a clinically important reduction in dissatisfaction with postpartum care (from $30 \%$ dissatisfied ${ }^{17}$ to $20 \%$ ).

\section{Recruitment}

Recruitment took place at one of Melbourne's three teaching hospitals from March 1996 to October 1998. Two research midwives (LD, AP) identified women who had had operative deliveries from the labour ward records and approached women on the postnatal ward at least 24 hours after the birth of their babies. We excluded women who had had stillbirths or babies weighing less than $1500 \mathrm{~g}$, women with insufficient English to take part, women who were ill or whose babies were ill, and women whose private obstetricians had refused permission to approach them.

A plain language information sheet about the study was provided to women, and written informed consent was obtained from all participants. Women completed a brief questionnaire providing sociodemographic data. Each midwife carried out recruitment and debriefing for half of each week's participants.

\section{Randomisation}

We used telephone randomisation to allocate women to debriefing or standard care, with allocation determined by separate computer generated, adaptive biased coin randomisation schedules for each research midwife.

Women allocated to standard care received a brief visit from the midwife to give them a pamphlet on sources of assistance for mothers on discharge from hospital. Women allocated to debriefing also received the pamphlet, and the midwife arranged a time for the debriefing session.

\section{Debriefing intervention}

The debriefing intervention provided women with an opportunity to discuss their labour, birth, and post-delivery events and experiences. Debriefing took place before the women were discharged from hospital. Both AP and LD are midwives experienced in talking with women about birth, able to listen with empathy to women's accounts, and aware of the common concerns and issues arising for women after an operative birth. Content of the discussion was determined by each woman's experiences and concerns, and up to one hour was made available for the session.

Each debriefing session was documented by the research midwife at the end of the session using a standard reporting sheet. The information recorded included duration of debriefing session, main issues and concerns raised by the woman, themes discussed, and support provided.

\section{Outcome assessment}

The primary outcomes were the prevalence of maternal depression at six months postpartum measured by the Edinburgh postnatal depression scale and overall maternal health status measured by the SF-36. These scales were assessed by a postal questionnaire sent to all participants. We chose to assess depression at six months because we had previously found raised rates of depression in the second half year after operative birth ${ }^{2}$ and because of other evidence of a high incidence of new cases of depression between the third and ninth month postpartum. ${ }^{18} 19$

The questionnaire also included detailed questions on satisfaction with care, experience of the birth, health problems, and (for women in the debriefing arm) opinion about the helpfulness of debriefing. The sequencing of the Edinburgh postnatal depression scale and the SF-36 was alternated in the questionnaires to reduce any completion order effect.

\section{Data management and analysis}

A coding schedule was developed by three of us (AP, JL, RS) with initial cross checking to ensure coding consistency. Questionnaires were then coded by one of us (AP). Data were double entered and validated. An intention to treat analysis was undertaken with SPSS $\mathrm{PC}+{ }^{20}$ and STATA $^{21}$ statistical packages. We used the recommended cut off for probable depression of 13 on the Edinburgh postnatal depression scale and assessed differences between the trial groups using odds ratios. Mean scores on the SF-36 subscales were compared by Student's $t$ test and 95\% confidence intervals. We compared women's views of their postpartum hospital care (ratings of statements about care on a seven point scale) using ordinal logistic regression to provide a cumulative odds ratio that indicated the degree of association between trial group and agreement with the statement over the whole scale. ${ }^{21}$

\section{Results}

\section{Recruitment and participation}

Figure 1 summarises recruitment and participation and table 1 outlines the reasons for non-participation. Response rates to the outcome questionnaire were high: $467 / 520(90 \%)$ women in the debriefing arm and $450 / 521(86 \%)$ in the standard care arm returned completed questionnaires (total $=917,88 \%$ of women recruited). The difference in response rates between 


\begin{tabular}{lr}
\hline Table 1 Reasons for non-participation in study \\
\begin{tabular}{lr} 
Reason for non-participation & $\begin{array}{c}\text { No (\%) of } \\
\text { women }\end{array}$ \\
\hline Not eligible: & $440(19.2)$ \\
\hline Insufficient English & $152(6.6)$ \\
\hline Low birthweight baby & $39(1.7)$ \\
\hline Baby unwell & $39(1.7)$ \\
\hline Woman unwell & $15(0.7)$ \\
\hline Stillbirth or neonatal death & \\
\hline Not approached: & $387(16.9)$ \\
\hline Doctor had declined study participation for all his or her \\
patients
\end{tabular} \\
\hline Going home too soon to take part & $376(16.4)$ \\
\hline Already discharged & $334(14.6)$ \\
\hline Unavailable or too busy & $145(6.3)$ \\
\hline Other reasons & $92(4.0)$ \\
\hline Declined invitation to participate & $275(12.0)$ \\
\hline Total & $2294(100)$ \\
\hline
\end{tabular}

the two groups was not significant (odds ratio $=1.39$, $95 \%$ confidence interval 0.94 to 2.07 ).

\section{Characteristics of study participants}

Table 2 shows that the characteristics of the two study groups were comparable after randomisation. Compared with non-respondents, women who returned the outcome questionnaire were more likely to be older, married, better educated, have higher family incomes, to speak English very well if English was not their first language, and to have private health insurance.

Telephone follow up of non-respondents identified eight women who reported problems with depression. Some of the eight had received treatment, including one woman who was readmitted to hospital with probable puerperal psychosis. Two of the eight were in the debriefing group and six in the standard care group.

\section{Maternal health outcomes}

Women allocated to debriefing were not less likely to score as depressed on the Edinburgh postnatal depression scale than women allocated to standard care (table 3). The odds of depression in the debriefing group were raised, although not significantly. The mean scores did not differ significantly between the groups $(t=1.17, \mathrm{P}=0.24)$.

Women were also asked whether "feeling depressed or very unhappy for more than a few days" had been a problem for them in the six months since the birth. Although a higher proportion of women in the debriefing group reported depression as a problem, the difference was not significant $(123 / 445$ $(28 \%)$ v $94 / 430 \quad(22 \%)$; odds ratio $=1.37,95 \%$ confidence interval 1.00 to 1.86 ).

Table 4 shows women's mean scores on the subscales of the SF-36. Women allocated to debriefing had poorer health status on seven of the eight subscales, although the difference was significant only for role functioning (emotional).

\section{Women's views about debriefing and postpartum care}

Women were positive about debriefing, with only 26/463 $(6 \%)$ rating the debriefing session as "unhelpful"; $200(43 \%)$ rated it as "very helpful" and $237(51 \%)$ as "helpful."

Participation in debriefing did not affect women's views of their postpartum hospital care. In response to the question "How satisfied are you overall with the care you and your baby received in hospital after the birth?" the proportion of women responding that they were "very happy" was similar in both groups (133/464 (29\%) debriefing $v$ 124/447 (28\%) standard care). Likewise only $5 \%$ (24/464) of women in the debriefing group were "very unhappy" with their care compared with $7 \%(31 / 447)$ in the standard care group. There were no significant differences in ratings on the seven point scale between the two groups (cumulative odds ratio $=0.97,95 \%$ confidence interval 0.71 to 1.26 ; see $B M / \mathrm{s}$ website for further details) or in responses to more specific statements about different aspects of postpartum hospital care.

\section{Effect of research midwife}

We also analysed the primary trial outcomes for each research midwife separately and found no differences. Analysing by research midwife also made no difference to women's views about helpfulness of the debriefing sessions or about postpartum hospital care.

\begin{tabular}{|c|c|c|}
\hline $\begin{array}{l}\text { Characteristic (Total No of respondents in } \\
\text { debriefing/standard care arms) }\end{array}$ & $\begin{array}{l}\text { No (\%) in debriefing } \\
\text { arm }\end{array}$ & $\begin{array}{l}\text { No }(\%) \text { in standard } \\
\text { care arm }\end{array}$ \\
\hline \multicolumn{3}{|l|}{ Method of operative birth (n=467/450): } \\
\hline Elective caesarean & $125(27)$ & $113(25)$ \\
\hline Emergency caesarean & $162(35)$ & $145(32)$ \\
\hline Forceps & $155(33)$ & $162(36)$ \\
\hline Vacuum extraction & $25(5)$ & $30(7)$ \\
\hline \multicolumn{3}{|l|}{ Parity $(n=467 / 449):$} \\
\hline Primiparous & $284(61)$ & $296(66)$ \\
\hline Multiparous & $183(39)$ & $153(34)$ \\
\hline \multicolumn{3}{|l|}{ Maternal age (years) $(\mathrm{n}=466 / 450)$ : } \\
\hline$<25$ & $45(10)$ & $53(12)$ \\
\hline $25-34$ & $296(64)$ & $275(61)$ \\
\hline$\geqslant 35$ & $125(27)$ & $122(27)$ \\
\hline \multicolumn{3}{|l|}{ Marital status $(\mathrm{n}=467 / 450)$ : } \\
\hline Married & $360(77)$ & $348(77)$ \\
\hline Cohabiting & $83(18)$ & $78(17)$ \\
\hline Separated, divorced, or widowed & $7(1)$ & $5(1)$ \\
\hline Single & $17(4)$ & $19(4)$ \\
\hline \multicolumn{3}{|l|}{ Secondary education $(\mathrm{n}=466 / 449)$ : } \\
\hline Completed year 12 & $306(66)$ & $304(68)$ \\
\hline Less than year 12 & $160(34)$ & $145(32)$ \\
\hline \multicolumn{3}{|l|}{ Tertiary education ( $\mathrm{n}=466 / 450)$ : } \\
\hline Degree or diploma & $244(52)$ & $223(50)$ \\
\hline Other or none & $222(48)$ & $227(50)$ \\
\hline \multicolumn{3}{|l|}{ English as first language $(n=467 / 449)$ : } \\
\hline Yes & $404(87)$ & $372(83)$ \\
\hline No & $63(13)$ & $77(17)$ \\
\hline \multicolumn{3}{|c|}{ English speaking ability if not first language $(\mathrm{n}=63 / 77)$ : } \\
\hline Very good & $50(79)$ & $55(75)$ \\
\hline Less than very good & $13(21)$ & $22(25)$ \\
\hline \multicolumn{3}{|l|}{ Family income $(\$ A)^{*}(n=444 / 427)$ : } \\
\hline$\leqslant 20000$ & $72(16)$ & $55(13)$ \\
\hline $20001-30000$ & $66(15)$ & $61(14)$ \\
\hline $30001-40000$ & $63(14)$ & $82(19)$ \\
\hline$>40000$ & $243(55)$ & $229(54)$ \\
\hline \multicolumn{3}{|l|}{ Pension or benefit main income $(n=465 / 447)$ : } \\
\hline Yes & $72(15)$ & $55(12)$ \\
\hline No & $393(85)$ & $392(88)$ \\
\hline \multicolumn{3}{|l|}{ Health insurance $(n=467 / 449)$ : } \\
\hline Public & $319(68)$ & $308(69)$ \\
\hline
\end{tabular}

\begin{tabular}{lll} 
Public & $319(68)$ & $308(69)$ \\
\hline Private & $148(32)$ & $141(31)$ \\
\hline$\$ 1-80.40$
\end{tabular}


Table 3 Edinburgh postnatal depression scores for debriefing and standard care groups

\begin{tabular}{lccccc} 
& Mean (SD) & $\begin{array}{c}\text { Median } \\
\text { (range) }\end{array}$ & $\begin{array}{c}\text { No }(\%) \\
\text { depressed } \\
\text { (score } \geqslant 13)\end{array}$ & $\begin{array}{c}\text { No }(\%) \text { not } \\
\text { depressed }\end{array}$ & Odds ratio (95\% Cl) \\
\hline Debriefing & $7.16(5.68)$ & $6.00(0-28)$ & $81(17)$ & $386(83)$ & $1.24(0.87$ to 1.77$)$ \\
\hline Standard care & $6.72(5.50)$ & $6.00(0-29)$ & $65(14)$ & $384(86)$ & 1.0 \\
\hline
\end{tabular}

Table 4 Mean (SD) SF-36 scores for debriefing and standard care groups

\begin{tabular}{lccc} 
SF-36 subscale & Debriefing & Standard care & $\boldsymbol{t}$ test (95\% Cl for difference) \\
\hline Physical functioning & $86.1(17.40)$ & $85.73(18.44)$ & $0.32(-1.96$ to 2.73$)$ \\
\hline Role functioning (physical) & $73.86(35.10)$ & $76.24(35.29)$ & $-1.02(-6.98$ to 2.22$)$ \\
\hline Bodily pain & $77.7(23.22)$ & $78.6(23.55)$ & $-0.59(-3.95$ to 2.13$)$ \\
\hline Mental health & $69.69(18.79)$ & $71.20(18.14)$ & $-1.23(-3.91$ to 0.89$)$ \\
\hline Role functioning (emotional) & $73.32(38.12)$ & $78.98(35.73)$ & $-2.31(-10.48$ to -0.84$)$ \\
\hline Vitality & $50.08(22.37)$ & $51.28(21.79)$ & $-0.82(-4.07$ to 1.68$)$ \\
\hline Social functioning & $78.78(24.28)$ & $80.47(23.69)$ & $-1.07(-4.80$ to 1.42$)$ \\
\hline General health & $72.20(20.91)$ & $73.22(21.00)$ & $-0.73(-3.75$ to 1.72$)$ \\
\hline
\end{tabular}

\section{Discussion}

This study contributes to the small number of randomised trials evaluating the effectiveness of debriefing in reducing mental health problems after traumatic events. It supports the systematic summary of current evidence by Wessely and others, which found no beneficial effect of debriefing. ${ }^{11}$

Nearly all women who experienced debriefing said that they found the session helpful. This finding was also reported in two other trials in which debriefing was found to be ineffective in reducing psychological problems. ${ }^{13} 14$ Not only did we find that health outcomes for the debriefing group were no better than those for women in the standard care group, we are not able to rule out the possibility that debriefing contributed to poorer emotional health. Women allocated to debriefing were more likely to report that depression had been a problem for them in the six months since the birth, and their SF-36 scores for emotional role functioning were significantly poorer. The nonsignificant differences on both the Edinburgh postnatal depression scale and the SF-36 were all in the direction of women in the debriefing group faring worse (with the exception of the SF-36 physical functioning subscale).

The UK trial that found a substantial benefit from midwifery led debriefing had several methodological problems. ${ }^{8}$ The study was designed to identify a reduction in the proportion of women scoring above seven on the hospital anxiety and depression scale (from $48 \%$ to $24 \%$ ), but the analysis was of the proportion of women scoring above 10 , for which the sample size planned (120) was below that required (292). In standard care more than half of women scored over 10 on both subscales, far more than in the pre-trial pilot. A "disappointment" factor may have increased the scores of women allocated to the control group. Outcome was assessed soon after hospital discharge, and the population was atypical: $59.6 \%$ were single women.

The idea that debriefing might be ineffective or even detrimental is not new. Some authors have raised the possibility of "secondary trauma" resulting from re-exposure to the experience during the debriefing session, ${ }^{10}{ }^{11}$ and a process of distancing the self from the experience has been suggested to be potentially adaptive ${ }^{13}$ Timing of the debriefing session, the people
What is already known on this topic

Operative birth has been associated with negative psychological sequelae for women, including depression, loss of self esteem, regret, guilt, and feelings of violation

Debriefing is widely used to reduce psychological disturbance after trauma despite little evidence of its effectiveness

Debriefing is being introduced for women after childbirth with the aim of improving psychological recovery

\section{What this study adds}

A midwife led debriefing session after an operative delivery did not reduce subsequent maternal depression, improve overall maternal health status, or increase satisfaction with postpartum care compared with standard care

The trend in all measures of psychological wellbeing was for women in the debriefing arm to fare worse, although the difference was significant for only one measure

The results do not support the introduction of routine debriefing after an operative delivery to improve maternal emotional health

involved, the presence or absence of other factors (personality traits, social support, and individual coping styles in response both to distress and to debriefing) may also be important in determining both psychological outcome after trauma and the potential effectiveness of debriefing. ${ }^{9-11} 2223$

Evidence from randomised trials is critical to the resolution of these issues. Ours was a pragmatic trial, and the intervention was designed to be incorporated within standard postpartum care if it proved effective. Debriefing was arranged during the postpartum hospital stay, and we used midwives as "debriefers" because of their predominant role in postpartum care. The use of midwives was also important given concerns that using mental health staff for debriefing might be detrimental, unnecessarily medicalising distress. ${ }^{24}$ Our findings, however, provide no evidence to recommend debriefing by midwives for women after operative birth as a strategy to reduce subsequent maternal depression or improve women's views of their postpartum care.

We thank all the women who participated in the trial, the nursing and medical staff at the participating hospital for their facilitation of the project, and Lyn Watson for statistical advice and assistance.

Contributors: RS drafted the grant application for the study, participated jointly in protocol design and discussion of core ideas at research team meetings, was the principal investigator for the study in its final two years, conducted the data analysis, and drafted the paper. JL initiated the study, formulated the research questions, contributed to the grant application, participated jointly in protocol design and discussion of core ideas at research team meetings, and contributed to the writing of the paper. LD contributed to the grant application and protocol design, undertook recruitment and conducted the debriefing intervention, participated in data collection and discussion of 
core ideas at research team meetings, and contributed to the paper. AP contributed to protocol design, undertook recruitment and conducted the debriefing intervention, participated in data collection and discussion of core ideas at research team meetings, coded all the questionnaires, and contributed to the paper. UW participated jointly in protocol design and discussion of core ideas at research team meetings, was the principal investigator for the study in its first two years, and contributed to the paper.

Funding: Research and development grants advisory committee of the Australian Commonwealth Department of Health, Housing, and Community Services.

Competing interests: None declared.

Brown S, Lumley J. Maternal health after childbirth: results of an Australian population based survey. Br J Obstet Gynaecol 1998;105:156-61.

2 Astbury J, Brown S, Lumley J, Small R. Birth events, birth experiences and social factors in depression after birth Aust J Public Health 1994;18 $176-84$

3 Hillan EM. Short-term morbidity associated with caesarean delivery. Birth 1992;19:190-4.

4 MacArthur C, Lewis M, Knox EG. Health after childbirth. Br J Obstet Gynaecol 1991;98:1193-204.

5 Fisher J, Stanley R Burrows G. Psychological adjustment to caesarean delivery: a review of the evidence. J Psychosom Obstet Gynaecol 1990;11: 91-106.

6 Elliott SA. Relationship between obstetric outcome and psychological measures in pregnancy and the postnatal year. J Reprod Inf Psycho 1984;2:18-32.

7 Ralph K, Alexander J. Borne under stress. Nursing Times 1994;90:28-30.

8 Lavender T, Walkinshaw SA. Can midwives reduce postpartum psychological morbidity? A randomized trial. Birth 1998;25:215-21.

9 Bisson JI, Deahl MP. Psychological debriefing and prevention of post-traumatic stress: more research is needed. $\mathrm{Br} J$ Psychiatry 1994:165:717-20.

10 Raphael B, Meldrum L. Does debriefing after psychological trauma work? BMJ 1995;310:1479-80
11 Wessely S, Rose S, Bisson J. A systematic review of brief psychological interventions ("debriefing") for the treatment of immediate trauma related symptoms and the prevention of post traumatic stress disorder. In: Cochrane Collaboration. Cochrane Library. Issue 4. Oxford: Update Software, 1999.

12 Bisson JI, Jenkins PL, Alexander J, Bannister C. Randomised controlled trial of psychological debriefing for victims of acute burn trauma. $\mathrm{BrJ}$ Psychiatry 1997;171:78-81.

13 Lee C, Slade P, Lygo V. The influence of psychological debriefing on emotional adaptation of women following early miscarriage: A preliminary study. Br J Med Psychol 1996;69:47-58.

14 Hagan R, Priest S, Evans S, Malmgren S, St Jack A, Henderson J, et al. Stress debriefing after childbirth: maternal outcomes [abstract A84]. In: Proceedings of the third annual congress of the Perinatal Society of Australia and New Zealand, March 1999. Parramatta, NSW: PSANZ, 1999:95.

15 Cox JL, Holden JM, Sagovsky R. Detection of postnatal depression: development of the 10-item Edinburgh postnatal depression scale. $\mathrm{Br}$ J Psychiatry 1987;150:782-6.

16 Ware JE, Sherbourne CD. The MOS 36-item short-form health survey (SF-36). 1. Conceptual framework and item selection. Med Care 1992;30:473-83.

17 Brown S, Lumley J, Small R, Astbury J. Missing voices. The experience of motherhood. Melbourne: Oxford University Press, 1994:101.

18 Nott PN. Extent, timing and persistence of emotional disorders following childbirth. Br J Psychiatry 1987;151:523-7.

19 O'Hara MW, Swain AM. Rates and risk of postpartum depression-a meta-analysis. Int Rev Psychiatry 1996;8:37-54

20 SPSS Incorporated. SPSS for windows, version 9.0. Chicago IL: SPSS, 1998

21 StataCorp. Stata statistical software; release 6.0. College Station, TX: Stata Corporation, 1999

22 Conlon L, Fahy TJ, Conroy R. PTSD in ambulant RTA victims: a randomized controlled trial of debriefing. J Psychosom Res 1999;46:37-44

23 Hobbs M, Mayou R, Harrison B, Worlock P. Randomised controlled tria of psychological debriefing for victims of road traffic accidents. $B M$ 1996;313:1438-9.

24 Wessely S. Commentary: reducing distress after normal childbirth. Birth 1998;25:220-1.

\title{
Randomised comparison of the effectiveness and costs of community and hospital based mental health services for children with behavioural disorders
}

\author{
Richard Harrington, Sarah Peters, Jonathan Green, Sarah Byford, Jane Woods, Ruth McGowan
}

\begin{abstract}
Objective To test the hypothesis that a community based intervention by secondary child and adolescent mental health services would be significantly more effective and less costly than a hospital based intervention.

Design Open study with two randomised parallel groups.

Setting Two health districts in the north of England.

Participants Parents of 3 to 10 year old children with behavioural disorder who had been referred to child and adolescent mental health services.

Intervention Parental education groups

Main outcome measures Parents' and teachers' reports of the child's behaviour, parental depression, parental criticism of the child, impact of the child's behaviour on the family.

Results 141 subjects were randomised to community $(\mathrm{n}=72)$ or hospital $(\mathrm{n}=69)$ treatment. Primary outcome data were obtained on $115(82 \%)$ cases a year later. Intention to treat analyses showed no significant differences between the community and hospital based groups on any of the outcome
\end{abstract}

measures, or on costs. Parental depression was common and predicted the child's outcome. Conclusions Location of child mental health services may be less important than the range of services that they provide, which should include effective treatment for parents' mental health problems.

\section{Introduction}

Views about where to provide secondary mental health services for children and adolescents have changed repeatedly over the past 50 years. Child psychiatry started as a community discipline in child guidance clinics. ${ }^{1}$ There were, however, many practical difficulties in the administration of these clinics, ${ }^{1}$ and during the 1970s and '80s many clinics closed and were replaced by hospital based services. ${ }^{12}$ Over the past 15 years, however, hospital based services too have been criticised-on the grounds that they are inaccessible, stigmatising, expensive, poorly integrated with community services, and less likely to produce gains that generalise to other environments, such as school. ${ }^{34}$ Political pressure has also been applied to child mental health services to return to the community.
Department of

Child and

Adolescen

Psychiatry,

University of

Manchester,

Manchester

M27 4HA

Richard Harrington

professor

Sarah Peters

research associate

Jonathan Green senior lecturer

Jane Woods

research assistant

Ruth McGowan

specialist registrar

continued over

BMJ 2000;321:1047-50

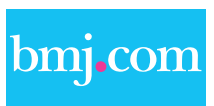

The full version of this paper appears on the BMJ's website 